University of Nebraska - Lincoln

DigitalCommons@University of Nebraska - Lincoln

Faculty Publications from the Department of Electrical and Computer Engineering

11-30-1996

Controlling three-axis attitude rates on a pointing system using nonlinear observer and two axis measurements

Marcelo C. Algrain

Follow this and additional works at: https://digitalcommons.unl.edu/electricalengineeringfacpub

Part of the Computer Engineering Commons, and the Electrical and Computer Engineering Commons

This Article is brought to you for free and open access by the Electrical \& Computer Engineering, Department of at DigitalCommons@University of Nebraska - Lincoln. It has been accepted for inclusion in Faculty Publications from the Department of Electrical and Computer Engineering by an authorized administrator of DigitalCommons@University of Nebraska - Lincoln. 


\section{Controlling three-axis attitude rates on a pointing system using nonlinear observer and two- axis measurements}

\author{
Marcelo C. Algrain, MEMBER SPIE \\ University of Nebraska-Lincoln \\ Department of Electrical Engineering and \\ Center for Electro-Optics \\ Lincoln, Nebraska 68588-0511 \\ E-mail: malgrain@unlinfo.unl.edu
}

\begin{abstract}
A new observer design method that allows for estimating the angular rates along a vehicle's three principal axes is described. The observer uses measurements from a single two-axis angular rate sensor (gyro) and determines the rates for the third axis using a nonlinear observer. Unlike conventional approaches where the equations governing vehicle motion (Euler's equations) are linearized and then an observer is constructed based on the linear model, this method does not require linearization of the system. Instead, a pseudo-linear representation is used. The pseudo-linear model is obtained by systematically decomposing a nonlinear system into linear and nonlinear terms. The nonlinear components are then redefined as an auxiliary set of state variables and/or inputs. This leads to an augmented linear system representation that is mathematically equivalent to the original nonlinear system. This method enables standard linear observer design techniques to be applied and develops observers that are capable of estimating the thirdaxis angular rates using measurements corresponding to the other two axes. The effectiveness of this approach is illustrated with an example. The case studied is the complete attitude rate determination and control of a spinning spacecraft. Computer simulation results show that the new approach provides excellent three-axis attitude control, yet requires angular rate sensors for only two axes. () 1997 Society of Photo-Optical Instrumentation Engineers. [S0091-3286(97)03004-3]
\end{abstract}

Subject terms: pointing and tracking system; attitude control; observer; feedback linearization.

Paper 24076 received July 24, 1996; revised manuscript received Nov. 28, 1996; accepted for publication Nov. 30, 1996.

\section{Introduction}

The angular motion of a vehicle in 3-D space contains a significant amount of cross-axis coupling information. In fact, the angular acceleration along one of the principal axes of inertia is proportional to the torque applied along that axis and proportional to the product of the angular velocities exerted along the other two axes orthogonal to the first. ${ }^{1}$ Thus, it is possible to determine the motion along one of the axes of rotation given measurements from the other two axes. This paper provides the means for accomplishing that objective. The benefit that this provides is that it may eliminate the need for a third sensor. Of course, this could lead to the obvious savings in sensor cost, but more importantly it provides a way to devise a fail-safe operating mode that may make it possible to overcome sensor failure, and/or it could be used as a powerful diagnostic tool for identifying such failures.

Since the cross-axis coupling effect is a nonlinear dynamical process, one cannot directly take data from two of the axes and calculate what is happening along the third. Thus, a parallel dynamic that mimics the original systems needs to be implemented. This is commonly known as designing an observer. This process is fairly straightforward for linear systems, ${ }^{2}$ but not so easily accomplished for nonlinear systems. ${ }^{3}$ Thus, a new observer design method is presented in this paper that facilitates its development.

To exploit the cross-axis coupling effect some amount of motion needs to be present for the coupling to occur. A good example of this would be a spin stabilized spacecraft. In this case, there is a continuous rotation along one of the axes (typically yaw). This would yield axis coupling and enable re-creation of the motion along one of the axes using an observer and measurements corresponding to the other axes. To gain more insight into the cross-axis coupling effect, consider the motion of a rigid body in three dimensions, which is described by the following functional equations ${ }^{4}$ :

$$
\Sigma F=m a, \quad \Sigma M_{O}=\dot{H}_{O},
$$

$$
\begin{aligned}
& \text { where } \\
& \qquad \begin{aligned}
\Sigma F & =\text { sum of forces acting on the body. } \\
m & =\text { mass of the rigid body } \\
a & =\text { acceleration } \\
\Sigma M_{O} & =\text { sum of moments around fixed point } O \\
\dot{H}_{O} & =\text { rate of change in angular momentum } H_{O} \\
& \text { about point } O .
\end{aligned}
\end{aligned}
$$


A considerable simplification is obtained in the general rotational equations of motion if the axes used to describe the motion are the principal axes of inertia. ${ }^{5}$ In such a case, the angular motion of the body is described by

$I_{1} \dot{\omega}_{1}+\left(I_{2}-I_{3}\right) \omega_{2} \omega_{3}=M_{1}$,

$I_{2} \dot{\omega}_{2}+\left(I_{1}-I_{3}\right) \omega_{1} \omega_{3}=M_{2}$,

$I_{3} \dot{\omega}_{3}+\left(I_{2}-I_{1}\right) \omega_{1} \omega_{2}=M_{3}$,

where $I_{1}, I_{2}$ and $I_{3}$ are the principal moments of inertia; $\omega_{1}$, $\omega_{2}$ and $\omega_{3}$ are components of the angular velocity vector $\omega$ along the principal axes; and $M_{1}, M_{2}$ and $M_{3}$ represent components of the moment vector $\mathbf{M}$ along the principal axes. Equations (2) are known as Euler's equations of motion. They provide a set of nonlinear differential equations that completely defines the angular motion of a rigid body in 3-D space. Although simple in form, these equations are nonlinear, making it difficult to design state observers using conventional design methods. For this reason, an equivalent representation of Euler's model is developed in the section that follows.

\section{Equivalent Pseudo-Linear Representation of Nonlinear Systems}

Many nonlinear system models can be converted into a mathematically equivalent expression that has a linear form. ${ }^{6}$ To see this development, consider a nonlinear system with the following general state-space form:

$$
\dot{\mathbf{X}}=\mathbf{A X}+\mathbf{f}(\mathbf{X})+\mathbf{g}(\mathbf{X}, \mathbf{U}), \quad \mathbf{Y}=\mathbf{C X},
$$

where

$$
\begin{aligned}
\mathbf{X}= & {\left[x_{1} x_{2} \ldots x_{n}\right]^{T}=\text { system state vector } } \\
\mathbf{U}= & \text { system input vector } \\
\mathbf{Y}= & \text { system output vector } \\
\mathbf{A}_{1}= & {\left[\mathbf{A}_{11}, \mathbf{A}_{12}, \ldots, \mathbf{A}_{1 n}\right]=\text { system matrix for linear } } \\
& \text { terms } \\
\mathbf{A}_{1 i}= & \text { row vector of constant coefficients with } \\
& i=1, \ldots, n \\
\mathbf{C}= & \text { measurement matrix } \\
\mathbf{f}= & {\left[f_{1} f_{2} \ldots f_{n}\right]^{T}=\text { vector of nonlinear operators } } \\
f_{i}= & \text { nonlinear operation on state vector } \mathbf{X} \text { with } \\
& i=1, \ldots, n \\
\mathbf{g}= & {\left[g_{1} g_{2} \ldots g_{n}\right]^{T}=\text { vector of nonlinear operators } } \\
g_{i}= & \text { nonlinear operation on state vector } \mathbf{X} \text { and/or } \\
& \text { input } \mathbf{U} \text { with } i=1, \ldots, n .
\end{aligned}
$$

The term $\mathbf{A X}$ in Eq. (3) contains linear operations within the nonlinear system, the term $\mathbf{f}(\mathbf{X})$ embodies nonlinear functions of the states, and the term $\mathbf{g}(\mathbf{X}, \mathbf{U})$ includes nonlinear combinations of the states and/or inputs. Then, the individual elements of the state vector are given by the following set of first-order differential equations:

$$
\begin{aligned}
& \dot{x}_{1}=\mathbf{A}_{11} \mathbf{X}+f_{1}(\mathbf{X})+g_{1}(\mathbf{X}, \mathbf{U}), \\
& \dot{x}_{2}=\mathbf{A}_{12} \mathbf{X}+f_{2}(\mathbf{X})+g_{2}(\mathbf{X}, \mathbf{U}),
\end{aligned}
$$

$\dot{x}_{n}=\mathbf{A}_{1 n} \mathbf{X}+f_{n}(\mathbf{X})+g_{n}(\mathbf{X}, \mathbf{U})$.

An augmented and mathematically equivalent state representation of the previous set of nonlinear differential equations can be obtained by introducing the following set of variables:

$a_{i} z_{i}=f_{i}(\mathbf{X}) \quad$ with $i=1, \ldots, n$,

$b_{i} \nu_{i}=g_{i}(\mathbf{X}, \mathbf{U}) \quad$ with $i=1, \ldots, n$,

$w_{i}=\dot{f}_{i}(\mathbf{X}) \quad$ with $i=1, \ldots, n$,

where $a_{i}$ is the constant coefficient for pseudo state variable $z_{i}$, and $b_{i}$ is the constant coefficient for pseudo input $v_{i}$. This yields the following expressions:

$\dot{x}_{1}=\mathbf{A}_{11} \mathbf{X}+a_{1} z_{1}+b_{1} v_{1}$,

$\dot{x}_{2}=\mathbf{A}_{12} \mathbf{X}+a_{2} z_{2}+b_{2} v_{2}$,

$\dot{x}_{n}=\mathbf{A}_{1 n} \mathbf{X}+a_{n} z_{n}+b_{n} v_{n}$,

$\dot{z}_{1}=w_{1}$

$\dot{z}_{2}=w_{2}$

$\vdots$

$\dot{z}_{n}=w_{n}$

The previous representation, written in state-space form, yields the following vector expression:

$\dot{\mathbf{X}}_{\mathrm{eq}}=\mathbf{A}_{\mathrm{eq}} \mathbf{X}_{\mathrm{eq}}+\mathbf{B}_{\mathrm{eq}} \mathbf{U}_{\mathrm{eq}}$,
$\mathbf{Y}=\mathbf{C}_{\mathrm{eq}} \mathbf{X}_{\mathrm{eq}}$,

where

$$
\begin{aligned}
& \mathbf{X}_{\mathrm{eq}}=[\mathbf{X Z}]^{T} \quad \text { with } \quad \mathbf{X}=\left[x_{1} \ldots x_{n}\right], \quad \mathbf{Z}=\left[z_{1} \ldots z_{n}\right], \\
& \mathbf{U}_{\mathrm{eq}}=[\mathbf{V W}]^{T} \text { with } \mathbf{V}=\left[v_{1} \ldots v_{n}\right], \quad \mathbf{W}=\left[w_{1} \ldots w_{n}\right], \\
& \mathbf{A}_{\mathrm{eq}}=\left[\begin{array}{cc}
\mathbf{A}_{1} & \mathbf{A}_{2} \\
{[0]} & {[0]}
\end{array}\right], \quad \mathbf{B}_{\mathrm{eq}}=\left[\begin{array}{cc}
\mathbf{B} & {[0]} \\
{[0]} & \mathbf{I}_{n}
\end{array}\right] \text {, } \\
& \mathbf{C}_{\mathrm{eq}}=\left[\begin{array}{cc}
\mathbf{C} & {[0]} \\
{[0]} & {[0]}
\end{array}\right], \\
& \mathbf{A}_{1}=\left[\mathbf{A}_{11}, \mathbf{A}_{12}, \ldots, \mathbf{A}_{1 n}\right], \\
& \mathbf{A}_{2}=\operatorname{diagonal}\left[a_{1}, a_{2}, \ldots, a_{n}\right] \text {, } \\
& \mathbf{B}=\operatorname{diagonal}\left[b_{1}, b_{2}, \ldots, b_{n}\right] \text {. }
\end{aligned}
$$




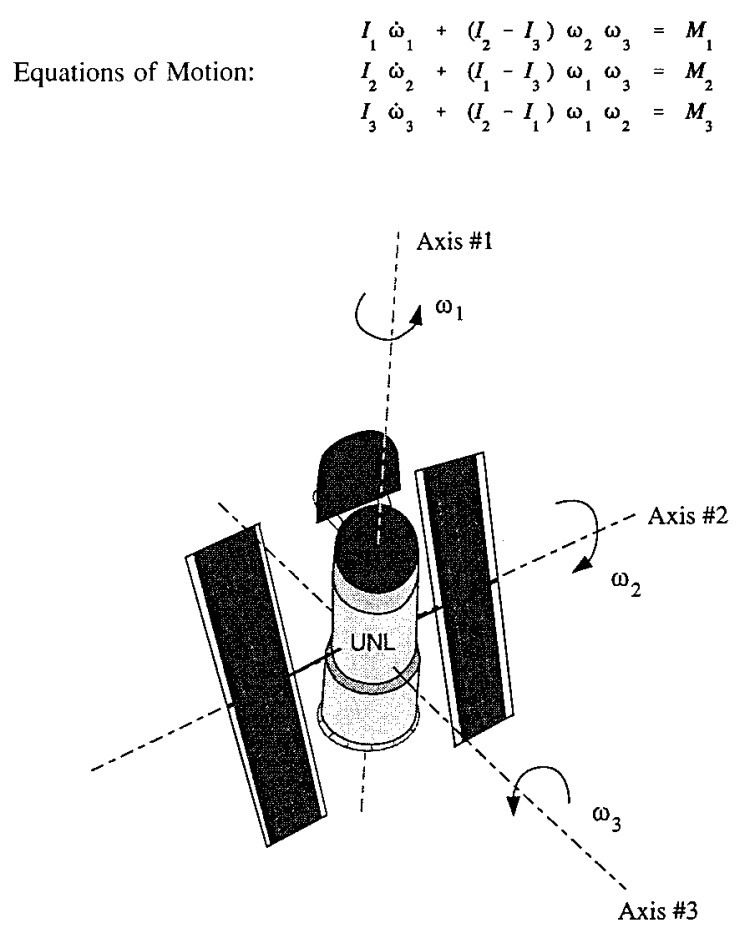

Fig. 1 Spacecraft undergoing rotational motion.

Equation (7) has a linear form in terms of the vectors $\mathbf{X}_{\mathrm{eq}}$ and $\mathbf{U}_{\text {eq }}$, and it is mathematically equivalent to the original nonlinear system. A restriction in this development is that the first-time derivative of the nonlinear function $f_{i}$ must exist for $i=1, \ldots, n$. The new state vector $\mathbf{X}_{\mathrm{eq}}$ contains the actual nonlinear system state variables $\mathbf{X}=\left[x_{1} \ldots x_{n}\right]^{T}$ and the pseudo state variables $\mathbf{Z}=\left[z_{1} \ldots z_{n}\right]^{T}$, which represent the system nonlinearities. The absence of nonlinear terms in some of the equations would reduce the number of auxiliary variables introduced, and the order of the augmented system would be less than $2 n$. Analogously to $\mathbf{X}_{\mathrm{eq}}$, the vector $\mathbf{U}_{\mathrm{eq}}$ is formed using pseudo inputs $v_{i}$, which result from the relationships in $g_{i}(\mathbf{X}, \mathbf{U})$. This variable substitution scheme leads to an augmented system that has a linear form and is mathematically equivalent to the original nonlinear system. This equivalent/pseudo-linear representation facilitates the application of linear system design methods, which are generally more powerful than their nonlinear counterparts. The term pseudo-linear is introduced to differentiate between a truly linear system and one that is artificially constructed through the introduction of new variables.

The particular case of interest in this paper is the control of 3-D angular spacecraft dynamics. The spacecraft is shown pictorially in Figure 1. Euler's Eqs. (2) describe its 3 -D angular motion. They can be converted into the equivalent pseudo-linear state-space model of Eq. (7) through the outlined augmentation method. Then, Euler's equivalent state-space model takes the following form:

$\dot{\omega}_{1}=a_{1} z_{1}+b_{1} \nu_{1}, \quad \nu_{1}=M_{1}$,

$\dot{\omega}_{2}=a_{2} z_{2}+b_{2} \nu_{2}, \quad \nu_{2}=M_{2}$,

$$
\begin{aligned}
& \dot{\omega}_{3}=a_{3} z_{3}+b_{3} \nu_{3}, \quad \nu_{3}=M_{3}, \\
& \dot{z}_{1}=w_{1}=\dot{\omega}_{2} \omega_{3}+\dot{\omega}_{3} \omega_{2}, \\
& \dot{z}_{2}=w_{2}=\dot{\omega}_{1} \omega_{3}+\dot{\omega}_{3} \omega_{1}, \\
& \dot{z}_{3}=w_{3}=\dot{\omega}_{1} \omega_{2}+\dot{\omega}_{2} \omega_{1} \\
& a_{1}=\frac{I_{2}-I_{3}}{I_{1}}, \quad a_{2}=\frac{I_{3}-I_{1}}{I_{2}}, \quad a_{3}=\frac{I_{1}-I_{2}}{I_{3}}, \\
& b_{1}=I_{1}^{-1}, \quad b_{2}=I_{2}^{-1}, \quad b_{3}=I_{3}^{-1} .
\end{aligned}
$$

The equivalent system matrices are given by

$$
\mathbf{A}_{\mathrm{eq}}=\left[\begin{array}{cccccc}
0 & 0 & 0 & a_{1} & 0 & 0 \\
0 & 0 & 0 & 0 & a_{2} & 0 \\
0 & 0 & 0 & 0 & 0 & a_{3} \\
0 & 0 & 0 & 0 & 0 & 0 \\
0 & 0 & 0 & 0 & 0 & 0 \\
0 & 0 & 0 & 0 & 0 & 0
\end{array}\right]
$$

$$
\mathbf{B}_{\mathrm{eq}}=\left[\begin{array}{cccccc}
b_{1} & 0 & 0 & 0 & 0 & 0 \\
0 & b_{2} & 0 & 0 & 0 & 0 \\
0 & 0 & b_{3} & 0 & 0 & 0 \\
0 & 0 & 0 & 1 & 0 & 0 \\
0 & 0 & 0 & 0 & 1 & 0 \\
0 & 0 & 0 & 0 & 0 & 1
\end{array}\right], \quad \mathbf{C}_{\mathrm{eq}}=\left[\begin{array}{cc}
\mathbf{C} & {[0]} \\
{[0]} & {[0]}
\end{array}\right] .
$$

This provides an equivalent linear representation that can be used in the design of state observers, on selection of a specific measurement matrix $\mathbf{C}$, as discussed in the next section.

\section{State Observer Design}

The reason for constructing an observer is to reproduce those states that are not available from measurements. Designing observers for linear systems is usually a straightforward process, for systems that are observable, ${ }^{7}$ but this is not so easily accomplished for nonlinear systems. Therefore, the development of a pseudo-linear equivalent system representation makes it more feasible to reconstruct the states of a nonlinear system based on available system outputs. ${ }^{8}$ This is because the nonlinear observer design can now be done using standard linear observer design methods, ${ }^{9}$ provided the pseudo-linear representation is state observable, i.e., the $\left[\mathbf{C}_{\mathrm{eq}}, \mathbf{C}_{\mathrm{eq}} \mathbf{A}_{\mathrm{eq}}, \ldots, \mathbf{C}_{\mathrm{eq}} \mathbf{A}_{\mathrm{eq}}^{n-1}\right]$ matrix has full rank. ${ }^{10}$ In such cases, the observer output reproduces the state vector, even though the system output provides an incomplete representation of this state vector.

This process is illustrated in Figure 2. The dynamics of the linear observer are defined as follows:

$\hat{\mathbf{X}}_{\mathrm{eq}}=\left(\mathbf{A}_{\mathrm{eq}}-\mathbf{L} \mathbf{C}_{\mathrm{eq}}\right) \hat{\mathbf{X}}_{\mathrm{eq}}+\mathbf{L Y}+\mathbf{B}_{\mathrm{eq}} \mathbf{U}_{\mathrm{eq}}$, 


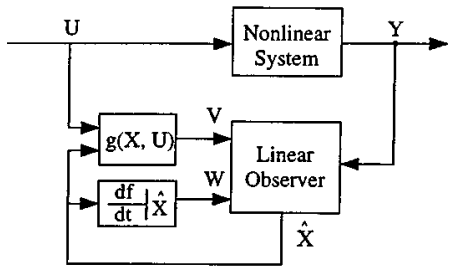

Fig. 2 State observer block diagram for nonlinear system.

$\begin{aligned} \hat{\mathbf{X}}_{\mathrm{eq}} & =[\hat{\mathbf{X}} \hat{\mathbf{Z}}]^{T}=\text { observer state vector } \\ \mathbf{Y} & =\text { system output vector, i.e., measured variables } \\ \mathbf{L} & =\text { matrix that yields the desired observer poles. }\end{aligned}$

The observer design consists in determining the matrix $\mathbf{L}$ so that the eigenvalues of the matrix $\left[\mathbf{A}_{\mathrm{eq}}-\mathbf{L} \mathbf{C}_{\mathrm{eq}}\right]$ are as specified. Even though the observer design is based on the pseudo-linear model, the state estimation for the nonlinear system is effectively accomplished when the pseudo-linear system is replaced with the original nonlinear system. This is because of the mathematical identity between the two systems. For generality, the full-order observer case is considered here, but the same design procedure applies to reduced-order observers, as discussed in the remainder of this section.

In practice, it is desirable to simplify the observer design by reproducing only those states that are not directly available from measurements. This is desirable because it simplifies the problem and reduces computational burden. The result is the so-called reduced-order observer. ${ }^{11}$ In this case, the states available from measurements are eliminated from the estimation problem, yielding a smaller number of states to be reproduced. In so doing, it is advantageous to rearrange the state vector so that the states measured appear as the top variables of the state vector, yielding the following partitioning of the state equations:

$$
\begin{aligned}
& \mathbf{X}_{\mathrm{eq}}=\left[\begin{array}{l}
\mathbf{X}_{1} \\
\mathbf{X}_{2}
\end{array}\right], \quad \mathbf{A}_{\mathrm{eq}}=\left[\begin{array}{ll}
\mathbf{A}_{11} & \mathbf{A}_{12} \\
\mathbf{A}_{21} & \mathbf{A}_{22}
\end{array}\right], \quad \mathbf{B}_{\mathrm{eq}}=\left[\begin{array}{l}
\mathbf{B}_{1} \\
\mathbf{B}_{2}
\end{array}\right], \\
& \mathbf{C}_{\mathrm{eq}}=[\mathbf{I}[0]],
\end{aligned}
$$

where $\mathbf{X}_{1}$ represents the states available from measurements and $\mathbf{X}_{2}$ the ones to be reproduced by the observer. Then, the reduced-order observer equations for the two portions of the partitioned state vector are

$\hat{\mathbf{X}}_{1}=\mathbf{Y}, \quad \hat{\mathbf{X}}_{2}=\mathbf{A}_{r} \hat{\mathbf{X}}_{2}+\mathbf{L}_{r} \mathbf{Y}_{r}+\mathbf{Z}_{r}$,

with

$\mathbf{A}_{r}=\mathbf{A}_{22}-\mathbf{L}_{r} \mathbf{A}_{12}$,

$\mathbf{Y}_{r}=\dot{\mathbf{X}}_{1}-\mathbf{A}_{11} \mathbf{X}_{1}-\mathbf{B}_{1} \mathbf{U}_{\mathrm{eq}}$

$\mathbf{Z}_{r}=\mathbf{A}_{21} \mathbf{X}_{1}+\mathbf{B}_{2} \mathbf{U}_{\mathrm{eq}}$

A block diagram depicting the observer implementation is shown in Figure 3. The reduced-order observer matrix

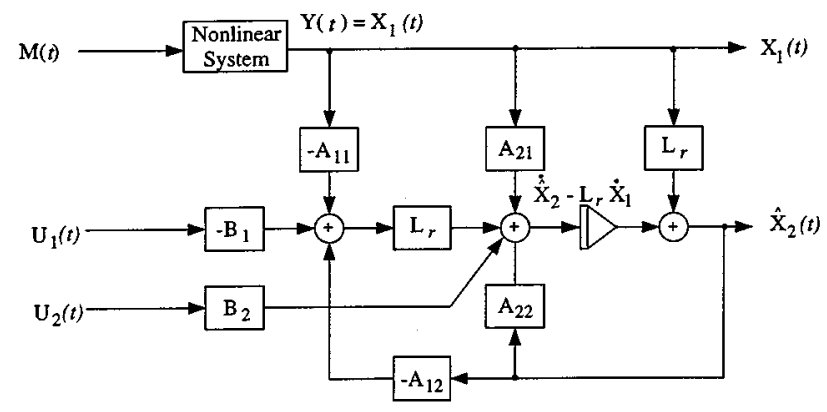

Fig. 3 Reduced-order observer block diagram.

can be computed in a similar manner as the $\mathbf{L}$ matrix for full-order observers. This is accomplished by solving for $\mathbf{L}_{r}$ in system of Eqs. (14), where $\lambda_{i}$ is an observer eigenvalue and $\boldsymbol{\psi}_{i}$ is an eigenvector associated with $\lambda_{i}$.

$\left[\left(\lambda_{i} \mathbf{I}-\mathbf{A}_{22}^{T}\right) \mathbf{A}_{12}^{T}\right]\left[\boldsymbol{\psi}_{i} \mathbf{L}_{r}^{T} \boldsymbol{\psi}_{i}\right]^{T}=[0]$

The preceding observer design methodology is applicable when the 3-D angular velocities of a vehicle are to be determined, but the rates for only two axes are measured. Without loss of generality, consider the case where the rates along axes 1 and 2 are measured, and the rates for the third axis are reproduced by an observer. Then the measurement matrix [Eq. (15)] results (note that variable $z_{3}$ is considered as measured since it is equal to the product between $\omega_{1}$ and $\omega_{2}$ which are measured individually):

$\mathbf{C}_{\mathrm{eq}}=\left[\begin{array}{cccccc}1 & 0 & 0 & 0 & 0 & 0 \\ 0 & 1 & 0 & 0 & 0 & 0 \\ 0 & 0 & 0 & 0 & 0 & 1\end{array}\right]$

Before the observer is designed, the observability of the equivalent system should be verified. In so doing, it is found that the rank of the matrix $\left[\mathbf{C}_{\text {eq }}, \mathbf{C}_{\text {eq }} \mathbf{A}_{\text {eq }}, \mathbf{C}_{\text {eq }} \mathbf{A}_{\text {eq }}^{2}, \mathbf{C}_{\text {eq }} \mathbf{A}_{\text {eq }}^{3}, \mathbf{C}_{\text {eq }} \mathbf{A}_{\text {eq }}^{4}, \mathbf{C}_{\text {eq }} \mathbf{A}_{\text {eq }}^{5}\right]$ is equal to 5 , i.e., not full. Thus, the equivalent system is not completely observable. However, a slight modification of the pseudolinear system model yields a second equivalent representation that is fully observable. This modification amounts to adding and subtracting the state $x_{3}$ to the equivalent model as follows:

Before: $\quad \dot{z}_{3}=w_{3}$ and $w_{3}=\dot{x}_{1} x_{2}+\dot{x}_{2} x_{1}$,

After: $\dot{z}_{3}=x_{3}+w_{3}^{\prime}$ and $w_{3}^{\prime}=w_{3}-x_{3}$.

This modification yields the following modified system matrix:

$\mathbf{A}_{\mathrm{eq} / \bmod }=\left[\begin{array}{cccccc}0 & 0 & 0 & a_{1} & 0 & 0 \\ 0 & 0 & 0 & 0 & a_{2} & 0 \\ 0 & 0 & 0 & 0 & 0 & a_{3} \\ 0 & 0 & 0 & 0 & 0 & 0 \\ 0 & 0 & 0 & 0 & 0 & 0 \\ 0 & 0 & 1 & 0 & 0 & 0\end{array}\right]$. 
Conducting the observability test on the modified equivalent system verifies that the modified equivalent system is fully observable. Therefore, an observer can be designed for Euler's equations using the modified model and standard linear design methods. For practical purposes, a reduced-order observer is preferred. Then, the modified equivalent system model for Euler's equations is partitioned as

$\mathbf{X}_{\text {mod }}=\left[\mathbf{X}_{1} \mathbf{X}_{2}\right]^{T}$ with $\quad \mathbf{X}_{1}=\left[x_{1} x_{2} z_{3}\right]$ and

$\mathbf{X}_{2}=\left[x_{3} z_{1} z_{2}\right]^{T}$,

$\mathbf{U}_{\text {mod }}=\left[\mathbf{U}_{1} \mathbf{U}_{2}\right]^{T}$ with $\quad \mathbf{U}_{1}=\left[v_{1} v_{2} w_{3}^{\prime}\right]$ and

$\mathbf{U}_{2}=\left[v_{3} w_{1} w_{2}\right]^{T}$,

where

$\mathbf{X}_{\text {mod }}=\left[\begin{array}{l}\mathbf{X}_{1} \\ \mathbf{X}_{2}\end{array}\right], \quad \mathbf{A}_{\text {mod }}=\left[\begin{array}{ll}\mathbf{A}_{11} & \mathbf{A}_{12} \\ \mathbf{A}_{21} & \mathbf{A}_{22}\end{array}\right], \quad \mathbf{B}_{\text {mod }}=\left[\begin{array}{l}\mathbf{B}_{1} \\ \mathbf{B}_{2}\end{array}\right]$,
$\mathbf{C}_{\text {mod }}=\left[\begin{array}{l}\mathbf{I}_{3 \times 3} \mathbf{0}_{3 \times 3}\end{array}\right]$,

$\mathbf{A}_{11}=\left[\begin{array}{lll}0 & 0 & 0 \\ 0 & 0 & 0 \\ 0 & 0 & 0\end{array}\right], \quad \mathbf{A}_{12}=\left[\begin{array}{ccc}0 & a_{1} & 0 \\ 0 & 0 & a_{2} \\ 1 & 0 & 0\end{array}\right]$,

$\mathbf{A}_{21}=\left[\begin{array}{ccc}0 & 0 & a_{3} \\ 0 & 0 & 0 \\ 0 & 0 & 0\end{array}\right], \quad \mathbf{A}_{22}=\left[\begin{array}{lll}0 & 0 & 0 \\ 0 & 0 & 0 \\ 0 & 0 & 0\end{array}\right]$,

$\mathbf{B}_{1}=\left[\begin{array}{cccccc}b_{1} & 0 & 0 & 0 & 0 & 0 \\ 0 & b_{2} & 0 & 0 & 0 & 0 \\ 0 & 0 & 1 & 0 & 0 & 0\end{array}\right]$,

$\mathbf{B}_{2}=\left[\begin{array}{cccccc}0 & 0 & 0 & b_{3} & 0 & 0 \\ 0 & 0 & 0 & 0 & 1 & 0 \\ 0 & 0 & 0 & 0 & 0 & 1\end{array}\right]$.

These matrices completely define the reduced-order observer in Eq. (12), which reproduces the third axis angular rates based on the rates measured from the other two axes orthogonal to it. Note that the reduced-order observer reproduces $\omega_{3}$ as well as $\omega_{1} \omega_{3}$ and $\omega_{2} \omega_{3}$; however, only $\hat{\omega}_{3}$ is of real interest since it is used, along with the measured angular velocities $\omega_{1}$ and $\omega_{2}$, to control the 3-D attitude of the spacecraft. A description of the control system accomplishing this task is given in Section 4, and a performance evaluation of the combined estimation and control problem is presented in Section 5 .

\section{Control System for Attitude Rate Regulation}

The design of the attitude controller regulating spacecraft 3 -D body angular rates is based on feedback linearization methods. ${ }^{12}$ The objective is for the angular rates to track a

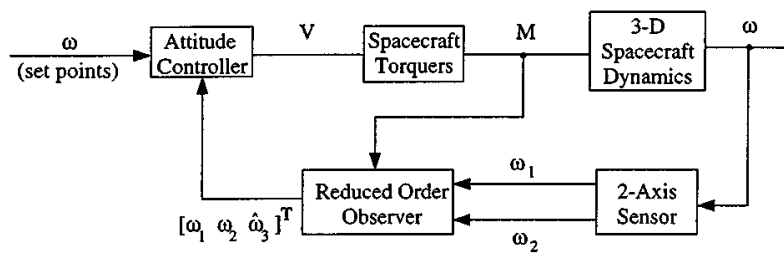

Fig. 4 Overall control system block diagram.

given setpoint profile. The system dynamics to be controlled are defined by Euler's Eqs. (2). Rewriting these equations in the form of Eq. (8) yields

$$
\begin{aligned}
& \dot{\omega}_{1}=a_{1} \omega_{2} \omega_{3}+b_{1} v_{1}, \\
& \dot{\omega}_{2}=a_{2} \omega_{1} \omega_{3}+b_{2} v_{2}, \\
& \dot{\omega}_{3}=a_{3} \omega_{1} \omega_{2}+b_{3} v_{3} .
\end{aligned}
$$

The control input $\mathbf{U}=\left[v_{1} v_{2} v_{3}\right]^{T}$ is designed so that the vehicle angular rates $\omega=\left[\omega_{1} \omega_{2} \omega_{3}\right]^{T}$ track the desired values $\omega_{\mathrm{SP}}=\left[\omega_{1 \mathrm{SP}} \omega_{2 \mathrm{SP}} \omega_{3 \mathrm{SP}}\right]^{T}$. Using feedback linearization techniques yields the following control laws:

$v_{1}=\frac{1}{b_{1}}\left[-a_{2} \omega_{2} \omega_{3}-k_{1}\left(\omega_{1}-\omega_{1 \mathrm{SP}}\right)+\dot{\omega}_{1 \mathrm{SP}}\right]$,

$v_{2}=\frac{1}{b_{2}}\left[-a_{2} \omega_{1} \omega_{3}-k_{2}\left(\omega_{2}-\omega_{2 \mathrm{SP}}\right)+\dot{\omega}_{2 \mathrm{SP}}\right]$,

$v_{3}=\frac{1}{b_{3}}\left[-a_{3} \omega_{1} \omega_{2}-k_{3}\left(\omega_{3}-\omega_{3 \mathrm{SP}}\right)+\dot{\omega}_{3 \mathrm{SP}}\right]$.

Substituting Eqs. (20) into Eqs. (19) and defining the error variables as

$e_{i}=\omega_{i}-\omega_{\mathrm{SP}} \quad$ with $i=1,2,3$,

yields the following equations for the error dynamics:

$\dot{e}_{i}+k_{i} e_{i}=0 \quad$ with $i=1,2,3$.

The dynamics of these equations are stable for any $k_{i}>0$ and $e_{i}$ tends to zero, or equivalently $\omega_{i}$ tends to $\omega_{i \mathrm{SP}}$ at the rate $e^{-k_{i} t}$. Therefore, the poles for the error dynamics are $\lambda_{i}=-k_{i}$ with $i=1,2,3$. This controller design uses all three system states in the linearization process. However, only two states are available from measurements $\left(\omega_{1}\right.$ and $\left.\omega_{2}\right)$. The third is provided by the reduced-order observer. This scenario constitutes a combined estimation/ linearization/control problem, which is schematically shown in Figure 4. For simplicity, the spacecraft torque actuators (thrusters, reaction wheels or torque rods) in Figure 4 are assumed to have unitary transfer functions. The effectiveness of this design is evaluated in Section 5 as part of a computer simulation case study.

\section{Computer Simulation Results}

The combined attitude rate estimation and control problem for a spacecraft is treated in this section. The spacecraft is modeled as a rigid body having the following principal mo- 


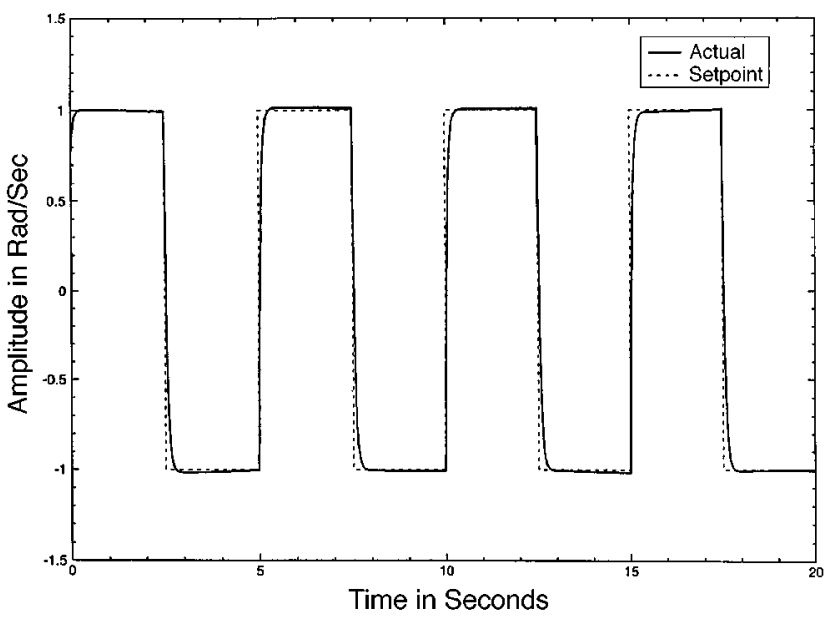

Fig. 5 Actual and desired angular rates for axis 1.

ments of inertia: $I_{1}=50 \mathrm{~kg} \mathrm{~m}^{2}, I_{2}=95 \mathrm{~kg} \mathrm{~m}^{2}$ and $I_{3}=110$ $\mathrm{kg} \mathrm{m}^{2}$. These values are representative of a midsize vehicle. The closed-loop system poles are arbitrarily selected to be equal to -0.5 , i.e., $k_{1}=k_{2}=k_{3}=0.5$. Also, the observer poles are arbitrarily selected to be equal to -1 . This determines, by solving Eq. (14), the following reduced-order observer matrix:

$\mathbf{L}_{r}=\left[\begin{array}{ccc}0 & 0 & 1 \\ -3.333 & 0 & 0 \\ 0 & 1.583 & 0\end{array}\right]$

The test scenario in this performance evaluation corresponds to the spacecraft undergoing an arbitrary maneuver in which the attitude control system is commanded to track the following setpoints:

- $\omega_{1 \mathrm{SP}}$ is a square wave with unit amplitude having a period of $5 \mathrm{~s}$.

- $\omega_{2 S P}$ is a triangular wave with unit amplitude having a period of $20 \mathrm{~s}$.

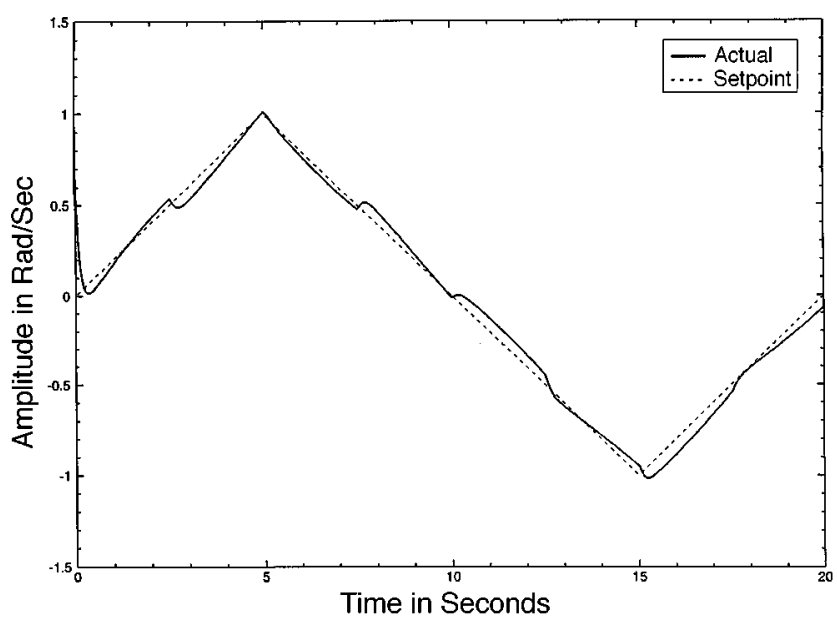

Fig. 6 Actual and desired angular rates for axis 2 .

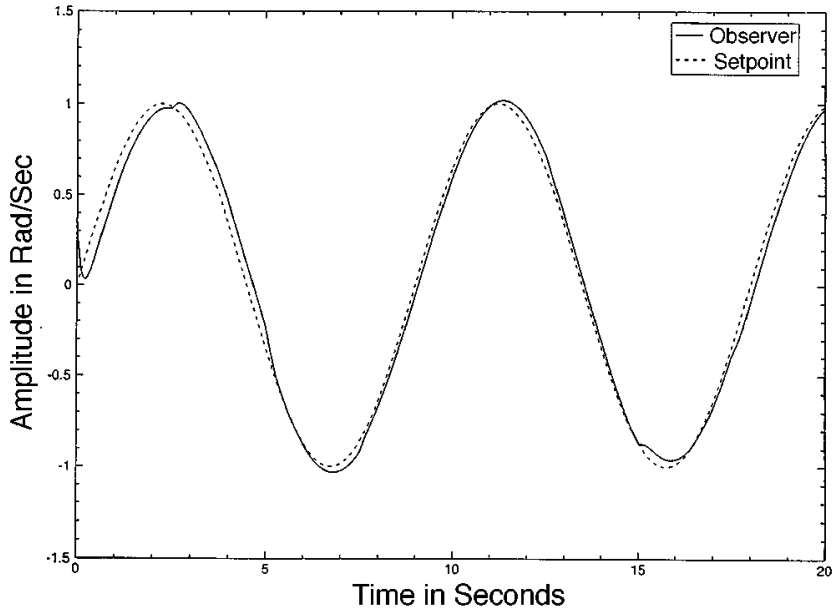

Fig. 7 Observer and desired angular rates for axis 3 .

- $\omega_{3 \mathrm{SP}}$ is a sinusoidal wave with unit amplitude having a period of $9 \mathrm{~s}$.

This maneuver may not be common, but it is used here to dramatize the effectiveness of the approach. Also, the initial attitude rates are assumed to be random and uniformly distributed between $\pm 1 \mathrm{rad} / \mathrm{s}$. The observer-assumed initial angular rate along the third axis is zero. Figure 5 shows the actual angular rate $\omega_{1}$ along axis 1 and the desired value

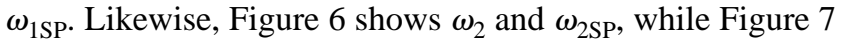
shows time plots for the desired angular rate $\omega_{3 \mathrm{SP}}$ and the rate $\omega_{3}$ estimated by the observer. In all instances, the controller does a good job at tracking the speed setpoints for each of the axes.

The performance of the observer is illustrated by Figure 8 , where the actual angular rate $\omega_{3}$ along the third principal axis is plotted versus time, as well as its observed value $\hat{\omega}_{3}$. It is seen from this figure that after only a few seconds, the angular rate reproduced by the observer tracks to the actual angular rate. Again, these accurate values for the third axis angular velocity are not directly measured, and they are estimated based on the measurements obtained from the other two axes orthogonal to it. This is important since it

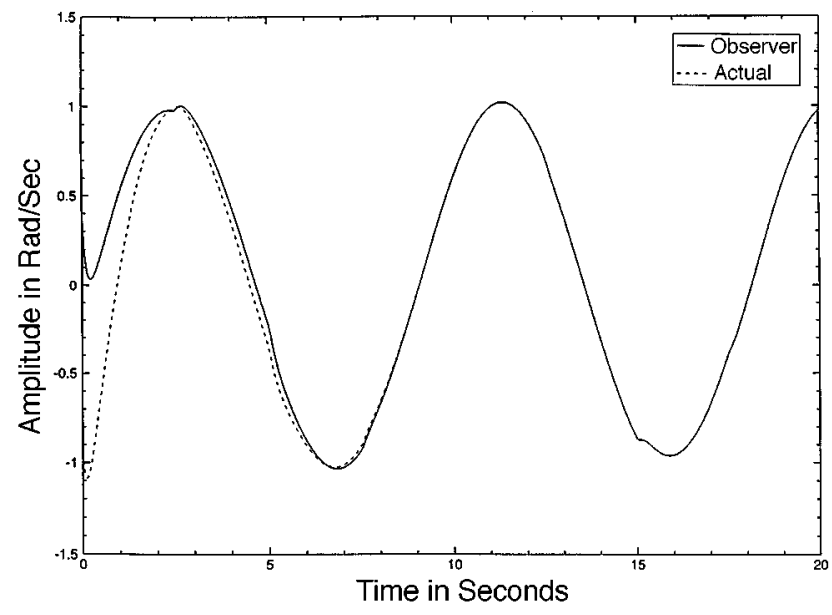

Fig. 8 Actual and observer angular rates for axis 3 . 
eliminates the need for a third axis sensor, which could lead to appreciable savings. Alternatively, all three axes could be fully instrumented and three observers could be constructed, each estimating the velocity of one axis using measurements from the other two, as the means to provide redundancy and/or diagnostic capabilities. Either way, the benefits are obvious.

\section{Summary and Conclusions}

This paper presents a novel observer design method applicable to nonlinear systems. The case studied is the 3-D angular rate determination and control for a spinning spacecraft. This approach enables the angular rates along one of the principal vehicle axes to be determined based on the measurements from the other two. The measured and observer reproduced body angular rates are used by a control system designed using feedback linearization methods. Computer simulation results show that the combined estimation and control problem is successfully accomplishedfor the spacecraft case study in this paper. Being able to construct an effective linear observer for a nonlinear case has far-reaching implications, including the possibility of eliminating a rate sensor from one of the axes, with the obvious savings consequences. Furthermore, if all three axes were instrumented, this method could be used as the means to provide redundancy and diagnostic capabilities, since an observer could be constructed for each of the three axes, each producing angular rates based on measurements corresponding to the other axes. Finally, this approach can also be applied to other nonlinear systems, providing a powerful and straightforward observer design method.

\section{References}

1. M. Algrain, "Gyroless line-of-sight stabilization for pointing and tracking systems," Opt. Eng. 33(4), 1255-1260 (1994),

2. D. Luenberger, "Observing the state of a linear system," IEEE Trans. Military Electron. MIL-8, 74-80 (Apr. 1964).
3. E. Misawa and J. Hedrick, "Nonlinear observers-a state-of-the-art survey," Trans. ASME 111, 344-352 (Sep. 1989).

4. F. Beer and E. Johnston, Section 18.1, Introduction, in Vector Mechanics for Engineers, 4th ed., McGraw-Hill, New York (1984).

5. A. D'Souza and V. Garg, Section 4.6.3, "State-variable formulation of the equations of motion," in Advanced Dynamics, Modeling and Analysis, Prentice-Hall, Englewood Cliffs, NJ (1984).

6. M. Algrain and D. Ehlers, "Novel Kalman filtering method for the suppression of gyroscope noise effects in pointing and tracking systems," Opt. Eng. 34(10), 3017-3030 (1995).

7. C. T. Chen, Section 5.4 in Linear System Theory and Design, Holt, Rinehart and Winston, New York (1984).

8. M. Algrain, "Design of observers and feedback controllers using pseudo-linear models of nonlinear systems," Proc. Am. Control Conf., June 1995, Seattle, WA, pp. 3714-3718.

9. T. Kailath, Section 4.1, "Asymptotic observers for state measurement," in Linear Systems, Prentice-Hall, Englewood Cliffs, NJ (1980).

10. B. Kuo, Section 5.11.1, "Definition of observability," in Automatic Control Systems, 7th ed., Prentice-Hall, Englewood Cliffs, NJ (1995).

11. W. Brogan, Section 13.6, "Observers,", in Modern Control Theory, 3rd ed., Prentice-Hall, Englewood Cliffs, NJ (1991).

12. J. Slotine and W. Li, "Feedback linearization," Chapter 6, in Applied Nonlinear Control, Prentice-Hall, Englewood Cliffs, NJ (1991).

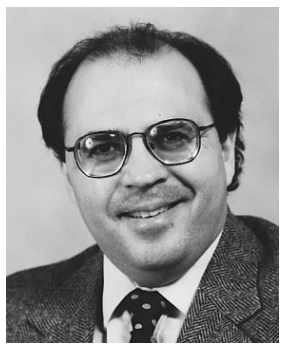

Marcelo C. Algrain received a BSME in 1980 and an MSEE in 1981, both from Wright State University, Dayton, Ohio, and a $\mathrm{PhD}$ in electrical engineering in 1991 from the Illinois Institute of Technology, Chicago. Since 1991 he has been an assistant professor at the University of Nebraska-Lincoln (UNL). From 1989 to 1991, he was an engineering specialist at Recon/Optical, Inc., where he performed analyses on stabilization and control systems for high-resolution reconnaissance systems. From 1984 to 1989, he was with Borg-Warner Research, developing control system prototypes for a variety of automotive and industrial products. Prior to his time with Borg-Warner, he was with Babcock \& Wilcox, designing process control systems for nuclear power plants. His research interests are stabilization, pointing and tracking systems, and in stochastic, optimal and adaptive controls. Dr. Algrain is a member SPIE, IEEE, and AIAA. He has published numerous papers in the control systems field and holds two patents. 